\title{
Case Report of Laparoscopic Treatment of Biliary Peritonitis Due to Double Organ Injury after Swallowing of Chicken Bone
}

\author{
Ludmil Marinov*1, Manol Kalniev ${ }^{2}$ and Ivan Cholashki ${ }^{3}$ \\ ${ }^{1}$ Consultant General and Laparoscopic surgery, Private Clinic "St Francois", France \\ ${ }^{2}$ Consultant Traumatology and General surgery, University Hospital, Bulgaria \\ ${ }^{3}$ Consultant of General Surgery, State Hospital of Vratsa, Bulgaria
}

Received: 眥: October 01, 2018; Published: 制: October 24, 2018

*Corresponding author: Ludmil Marinov, Consultant General and Laparoscopic surgery, Private Clinic "St Francois”, Chartres, France

\begin{abstract}
A 52 years old woman presented to emergency service after eating chicken meat few days before that. She had upper abdominal pain, vomiting, weakness and fever of $38 \mathrm{oC}$. A control abdominal CT scan showed linear $50 \mathrm{~mm}$ foreign body that perforated the pyloric wall and penetrated into the liver. US, CT and fibro gastroscopy have been used to discover cause for the patient's problem. Laparoscopy was suggested as main therapeutic method for extraction of the foreign body and closing of organs damage
\end{abstract}

Keywords: Foreign Body; Injury; Biliary Leak; Peritonitis; Laparoscopy

Abbreviations: HB: Hemoglobin; HCT: Hematocrit; WBC: White Blood Cells; ASAT: Aspartate Transaminase; LDH: Lactate Dehydrogenase; ALP: Alkaline Phosphatase; $\gamma$ GT: $\Gamma$-Glutamic Transferase

\section{Introduction}

Accidental swallowing of foreign body is very rare and ends with egestion without medical observation and diet. Mostly, it is observed in patients at younger age [1]. However, when eating chicken, fish or crowing it can happen to patients of all ages. Sharper and longer than the diameter of the gastrointestinal tube bodies may lead to mechanical obstruction or perforate the GI wall causing local inflammation. In case of double organ injury, a sharp foreign body may cause life-threatening complication as liver abscess [2], bile leak, peritonitis or abdominal hemorrhage. CT imaging is the method for diagnostic and laparoscopy is preferred for dealing with abdominal complications [1-3]. The aim of this report is to present the importance of the laparoscopic surgery for the treatment of minimal injury of the gastrointestinal tract.

\section{Case Report}

A 52-year old female presented in emergency room for acute continuous abdominal pain located at epigastrium and right subcostal area since 4 days. One hour before examination, she felt acute pain in the upper abdomen, followed by multiple vomiting, fever of $38.5^{\circ} \mathrm{C}$ and asthenia. Antecedent's morbidity includes duodenal ulcer with positive test for Helicobacter pillory. It was treated with $\mathrm{H} 2$ blocker-Quamatel and Clarithromycin. No history of non-steroidal drug use. At physical examination, the patient presented with stable cardiopulmonary signs with respiratory rate $17 \mathrm{bpm}$, heart rate 76/min and blood pressure 110/84 mmHg. Abdominal wall revealed tenderness to palpation on the epigastrium and right subcostal quadrant, with positive sign of defense. Blood count analysis showed: Hemoglobin (Hb)-102 g/l; Erythrocytes(Ery)-2.24; Hematocrit (Hct)-0.21, White blood cells count (WBC) $15.0 \times 109 / \mathrm{L}$; Lymphocytes (Ly) $12 \times 109 / \mathrm{L}$; Neutrophils (Neu) $79 \times 109 / \mathrm{L}$; an erythrocyte sedimentation of 59 $\mathrm{mm} / \mathrm{h}$ and C-reactive protein (CRP) $145 \mathrm{mg} / \mathrm{L}$. Liver test results at upper levels: Aspartate transaminase (ASAT): 53U/L, Alanine aminotransferase (ALAT):62U/L, Lactate dehydrogenase (LDH) 456U/L. Alkaline phosphatase (ALP), $\gamma$-Glutamic transferase $(\gamma \mathrm{GT})$ and bilirubin are normal.

At time of admission, chest and abdominal radiographs had no pathologies. Abdominal ultrasonography examination revealed small quantity of free liquid collection. CT scan showed thin sharp foreign body $(52 \mathrm{~mm})$ that extends from prepiloric stomach wall 
to the left liver lobe (Figure 1). visual observation of the stomach using upper gastrointestinal endoscopy does not help to discover any pathology. The patient underwent diagnostic laparoscopy. Sharp, foreign body that perforated the stomach wall at prepyloric area, penetrated the liver causing fissure with hemorrhagia and biliorhagia, and forming local bilio peritonitis, was found (Figure 2). A $140 \mathrm{ml}$ of bile was aspirated. After diagnosis, the foreign body was slowly removed inferomedially from the liver and the stomach wall and extracted by $10 \mathrm{~mm}$ trocar. The perforated stomach was saturated with single separated sutures. Liver fissure was closed by single absorbable suture. Rinsing, aspiration and placement of tube drain finalized the operation (Figure 3). Despite the negative microbiology, the patient was treated postoperatively with Vancomycin. At day 1 the drain was removed, and the patient was discharged from the clinic at day 2. Control abdominal TDM, which was normal, was performed 10 days after the operation.

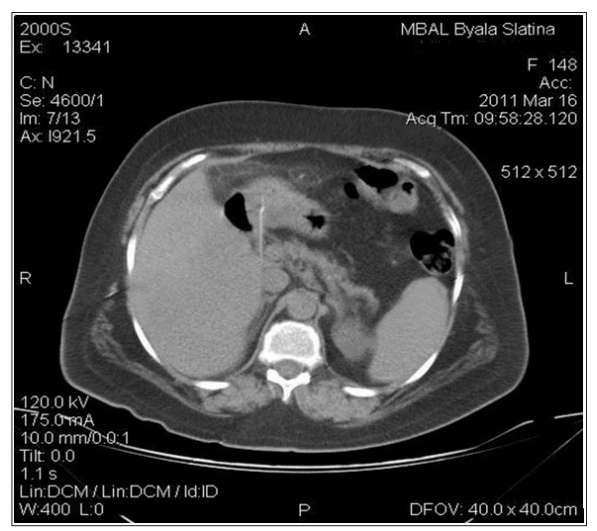

Figure 1: CT abdomen- foreign body in liver hilum.

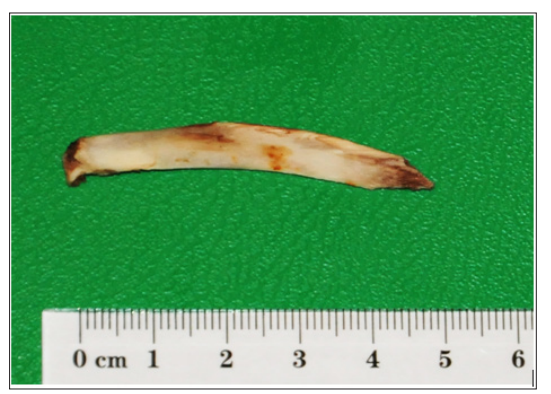

Figure 2: Chicken bone, causing double perforation, extracted by laparoscopy.

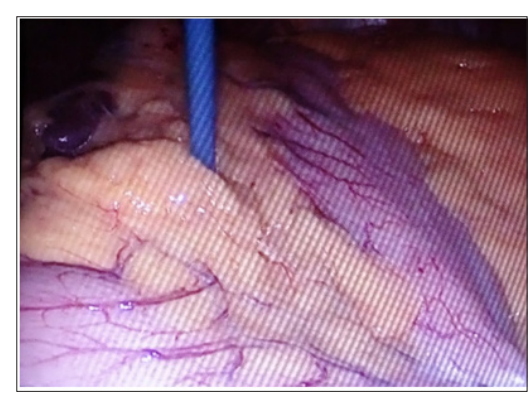

Figure 3: Prepyloric location of perforation.

\section{Discussion}

Swallowing of a foreign body is incidental and happens mostly to children and rarely to adults. Depending on its size and form, it usually passes out spontaneously. However, the thin and sharp bodies may turn transversally to the gastrointestinal wall causing impaction and perforation [1-3]. Unwanted type is observed in children when they play with small objects or consume non-selected chicken or fish meat. In adults it is accidentally, by consuming food with small parts of bone or being dental or otorhinolaryngology patients, workers in carpentry, dressmakers and circus artists [4] The second, willful swallowing of a foreign body is observed in criminals at prison and patients in psychiatric clinics [5] To provoke change of conditions the prisoners take multiple times self-prepared metallic bodies such as wire stars or wire hedgehog. Passing the upper gastrointestinal tract organs, these objects cause disruption of organ wall with life threatening complications. Clinic induced by foreign body may be asymptomatic if pass out spontaneously in $90 \%$. Only (1\%) of cases induce troubles that require emergency diagnosis and operative procedure.

Penetration of the stomach and duodenal wall may induce injuries of surrounding organ as liver and causing liver abscess, bilioma or biliary peritonitis [1-3,5]. Gastrointestinal discomforts with septic fever are principals. Diagnosis includes native abdominal X-ray radiography, CT and ultrasound. CT imaging is a more sensitive method and may show non-metallic foreign bodies as calcified chicken or fish bones, pneumoperitoneum after tissues covering of perforation, local fatty infiltration and intraabdominal liquid collection [5]. Different endoscopic methods are proposed after extraction: titanium clip placement, injection of sclerosing liquid and coagulation to stop bleeding or closing perforation $[7,8]$. Surgical treatment is required for cases with lumen obstruction, laceration with bleeding, and intraabdominal inflammation as abscess, peritonitis or hemorrhage. Laparoscopy is the method of choice for all abdominal complications caused by penetrating body. It permits to find the end of the body that causes perforation, to confirm an abdominal inflammation such as abscess or local peritonitis and to treat with removing, aspiration, irrigation, suture and draining.

\section{Conclusion}

Gastrointestinal complications after swallowing of food containing animal meet are not rare observed causes for abdominal emergency surgery. Mostly this is accidentally. Usually small parts of bones pass without clinical symptoms, but the once with sharp edges may cause perforation and periorgan local inflammatory processes or late abscess and peritonitis. CT scan is presented as the most useful diagnostic imaging method for study of abdominal organ pathology. Introduction of laparoscopy helps to minimize the surgical procedures for extraction of foreign bodies and treatment of surgical complications.

\section{References}

1. Santos SA, Alberto SC, Cruz E, Pires E, Figueira T, et al. (2002) Hepatic abscess induced by foreign body: case report and literature review. World J Gastroenterol 13(9): 1466-1470. 
2. Cheung YC, Ng SH, Tan CF, Ng KK, Wan YL, et al. (2000) Hepatic inflammatory mass secondary to toothpick perforation of the stomach: triphasic CT appearances. Clin Imaging 24(2): 93-95.

3. Broome CJ, Peck RJ (2000) Hepatic abscess complicating foreign body perforation of the gastric antrum: an ultrasound diagnosis.Clin Radiol 55: 242-243.

4. Ugenti I, Digennaro R, Martines G, Caputi Iambrenghi O (2015) Double esophageal perforation by ingested foreign body: Endoscopic and surgical approach. A case report. Int J Surg Case Rep 17: 55-57.

5. Pinero Madrona A, Fernández Hernández JA, Carrasco Prats M, Riquelme Riquelme J, Parrila Paricio P, et al. (2000) Intestinal perforation by foreign bodies. Eur J Surg 166(4): 307-309.

ISSN: 2574-1241

DOI: 10.26717/BJSTR.2018.10.001940

Ludmil Marinov. Biomed J Sci \& Tech Res

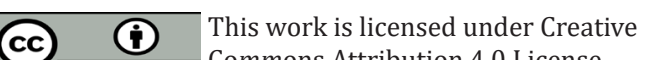

Submission Link: https://biomedres.us/submit-manuscript.php
6. Omejc M (2002) Laparoscopic removal of an ingested pin migrating into the liver. Surg Endosc 16 (3): 537.

7. Mukkada RJ, Chettupuzha AP, Francis VJ, Mathew PG, Chirayath SP et al. (2007) Endoscopic removal of chicken bone that caused gastric perforation and liver abscess. Indian J Gastroenterol 26(5): 246-247.

8. Kim JS, Kim HK, Cho YS, Chae HS, Kim CW, et al. (2008) Extraction and clipping repair of a chicken bone penetrating the gastric wall. World J Gastroenterol 14(12): 1955-1957.

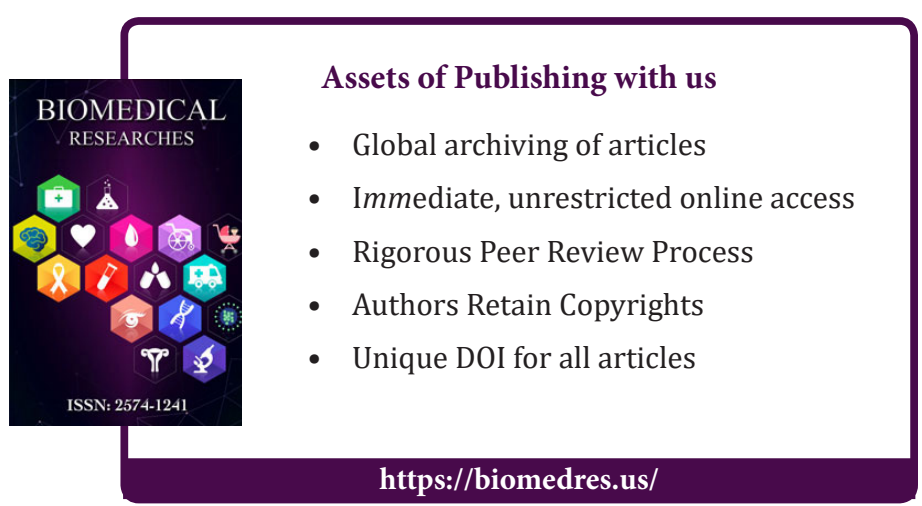

